OPEN ACCESS

Edited by: Kelvin Y. Kwan,

Rutgers University, The State University of New Jersey,

United States

Reviewed by:

Robert Lyle,

University of Oslo, Norway

Aishwarya Swaminathan,

University of Massachusetts Medical

School, United States

*Correspondence:

Ronna Hertzano

rhertzano@som.umaryland.edu

Specialty section:

This article was submitted to

Epigenomics and Epigenetics,

a section of the journal

Frontiers in Cell and Developmental

Biology

Received: 27 January 2018 Accepted: 13 April 2018

Published: 01 May 2018

Citation:

Matern MS, Beirl A, Ogawa Y, Song Y, Paladugu N, Kindt KS and Hertzano $R$

(2018) Transcriptomic Profiling of

Zebrafish Hair Cells Using RiboTag.

Front. Cell Dev. Biol. 6:47.

doi: 10.3389/fcell.2018.00047

\section{Transcriptomic Profiling of Zebrafish Hair Cells Using RiboTag}

\author{
Maggie S. Matern ${ }^{1}$, Alisha Beirl ${ }^{2}$, Yoko Ogawa ${ }^{1}$, Yang Song ${ }^{3}$, Nikhil Paladugu ${ }^{1}$, \\ Katie S. Kindt ${ }^{2}$ and Ronna Hertzano ${ }^{1,3,4 *}$
}

1 Department of Otorhinolaryngology Head and Neck Surgery, University of Maryland School of Medicine, Baltimore, MD, United States, ${ }^{2}$ Section on Sensory Cell Development and Function, National Institute on Deafness and Other Communication Disorders, Bethesda, MD, United States, ${ }^{3}$ Institute for Genome Sciences, University of Maryland School of Medicine, Baltimore, MD, United States, ${ }^{4}$ Department of Anatomy and Neurobiology, University of Maryland School of Medicine, Baltimore, MD, United States

The zebrafish inner ear organs and lateral line neuromasts are comprised of a variety of cell types, including mechanosensitive hair cells. Zebrafish hair cells are evolutionarily homologous to mammalian hair cells, and have been particularly useful for studying normal hair cell development and function. However, the relative scarcity of hair cells within these complex organs, as well as the difficulty of fine dissection at early developmental time points, makes hair cell-specific gene expression profiling technically challenging. Cell sorting methods, as well as single-cell RNA-Seq, have proved to be very informative in studying hair cell-specific gene expression. However, these methods require that tissues are dissociated, the processing for which can lead to changes in gene expression prior to RNA extraction. To bypass this problem, we have developed a transgenic zebrafish model to evaluate the translatome of the inner ear and lateral line hair cells in their native tissue environment; the Tg(myo6b:RiboTag) zebrafish. This model expresses both GFP and a hemagglutinin (HA) tagged rp/10a gene under control of the myo6b promoter (myo6b:GFP-2A-rp/10a-3xHA), resulting in HA-tagged ribosomes expressed specifically in hair cells. Consequently, intact zebrafish larvae can be used to enrich for actively translated hair cell mRNA via an immunoprecipitation protocol using an antibody for the HA-tag (similar to the RiboTag mice). We demonstrate that this model can be used to reliably enrich for actively translated zebrafish hair cell mRNA. Additionally, we perform a global hair cell translatome analysis using RNA-Seq and show enrichment of known hair cell expressed transcripts and depletion of non-hair cell expressed transcripts in the immunoprecipitated material compared with mRNA extracted from whole fish (input). Our results show that our model can identify novel hair cell expressed genes in intact zebrafish, without inducing changes to gene expression that result from tissue dissociation and delays during cell sorting. Overall, we believe that this model will be highly useful for studying changes in zebrafish hair cell-specific gene expression in response to developmental progression, mutations, as well as hair cell damage by noise or ototoxic drug exposure.

Keywords: inner ear, hair cells, zebrafish, RiboTag, RNA-Seq 


\section{INTRODUCTION}

Hearing loss is a genetically heterogeneous disorder, with mutations in over 150 genes estimated to underlie genetic nonsyndromic hearing deficits (Van Camp and Smith, 2017). Of these, a large proportion affect genes that are preferentially expressed in the sensory cells of the inner ear, namely the mechanosensory hair cells (HCs) (Elkon et al., 2015). Zebrafish have served as an excellent model system for functional analysis of genes in HC function, as they possess HCs both in the inner ear as well as within an external lateral line system, are easy to manipulate genetically, and generate large numbers of progeny within a short gestational period (Nicolson, 2005, 2017; Erickson and Nicolson, 2015). However, the study of the molecular changes induced by manipulation of HC-expressed genes in zebrafish has been limited due to a paucity of models that allow cell type-specific molecular analysis of changes in gene expression. Specifically, across vertebrate species, the auditory, vestibular, and lateral line sensory organs are comprised of a variety of cell types, of which HCs make up only a small percentage (Hertzano and Elkon, 2012; Jiang et al., 2014; Matern et al., 2017). Therefore, due to their relative scarcity, cell typespecific approaches such as manual cell sorting, fluorescence activated cell sorting (FACS), or single cell RNA-Seq (scRNASeq) are necessary to analyze gene expression in HCs. These methods have been used in both mice and zebrafish to analyze $\mathrm{HC}$ gene expression changes that occur in mutant animals, during development and regeneration, or after exposure to noise or ototoxic drugs (McDermott et al., 2007; Hertzano and Elkon, 2012; Jiang et al., 2014; Steiner et al., 2014; Burns et al., 2015; Elkon et al., 2015; Scheffer et al., 2015). However, both scRNASeq and cell sorting-based techniques require dissociation of tissues to obtain a single cell suspension. Tissue dissociation can induce significant cellular stress due to loss of lateral inhibition and cell-cell contact, and combined with the prolonged time associated with tissue processing, may lead to confounding changes in gene expression (Sanz et al., 2009; Gay et al., 2013, 2014).

To avoid dissociation-induced molecular changes, recent studies have developed techniques in both mice and zebrafish models to extract RNA from specific cell types within intact organs (Heiman et al., 2009; Sanz et al., 2009; Gay et al., 2013; Tryon et al., 2013; Erickson and Nicolson, 2015; Roh et al., 2017). These approaches rely on pulldown of RNA from a cell type of interest via tagged ribosomes, or directly tagged RNA. As an example, the RiboTag mouse model expresses a component of the $60 \mathrm{~S}$ subunit of the ribosome with a Cterminal hemagglutinin tag (RPL22-HA) (Sanz et al., 2009). Using this model, Cre-induced expression of RPL22-HA can be used to capture actively translated RNA from a cell type of interest via immunoprecipitation. The BACarray and NuTRAP mice models work in a similar way to RiboTag, however ribosomes are tagged with a green fluorescent protein (GFP). Additionally, the NuTRAP mice co-express nuclear tagging proteins that allow for concomitant epigenetic profiling of a Cre expressing cell type. In 2013, Tryon et al. also introduced several RiboTag/TRAP models to study cell type-specific gene expression in zebrafish (Tryon et al., 2013). Similar to the BACarray and NuTRAP mouse models, these zebrafish models rely on tagging the $60 \mathrm{~S}$ ribosomal subunit through non-inducible tissuespecific expression of an rpl10a-GFP fusion gene. Collectively, in mouse and zebrafish, RiboTag, BACarray, and NuTRAP can be used for cell type-specific isolation of RNA via pulldown of labeled ribosomes. In these models, the immunoprecipitated RNA is enriched for actively translated transcripts, and is referred to as the "translatome" rather than the whole cellular transcriptome.

An alternative method to ribosomal pulldown is to isolate cell type-specific RNA from a complex tissue environment by thiouracil (TU) tagging. This method relies on tissue specific expression of uracil phosphoribosyltransferase (UPRT), an enzyme capable of integrating 4-thiouracil into newly synthesized RNA, which can then be affinity purified. Both mice and zebrafish TU-tagging models have been developed, and rather than obtaining only actively translated RNA as in the RiboTag/TRAP models, this method allows for capture of all newly synthesized RNA within a cell type of interest (i.e., the transcriptome) after the application of 4-thiouracil (Gay et al., 2013, 2014; Erickson and Nicolson, 2015). As with the ribosomal pulldown techniques, affinity purified RNA is enriched for the cell type of interest, and also contains some RNA from other tissues. In zebrafish, TU-tagging has been used to identify HC expressed transcripts. However, this model was only able to identify a small number of genes, and only those genes with very high expression levels in HCs were found to be significantly enriched in the immunoprecipitated RNA compared to input (Erickson and Nicolson, 2015).

To more effectively isolate RNA from zebrafish HCs, we have developed a transgenic zebrafish RiboTag model to evaluate the translatome of zebrafish inner ear and lateral line HCs; the $T g$ (myo6b:GFP-2A-rpl10a-3xHA) zebrafish [from here on referred to as $T g(m y o 6 b$ :RiboTag)]. This model carries a construct to drive expression of both an HA-tagged Rpl10a protein and GFP in HCs under control of the HC-specific myo6b promoter (Seiler et al., 2004; Obholzer et al., 2008). To our knowledge, this is the first zebrafish model to allow for HCspecific gene expression analysis via two methods: (1) tissue dissociation and cell sorting based on GFP expression, and (2) immunoprecipitation of HA-tagged ribosomes to enrich for HC expressed transcripts. We use both RT-qPCR and RNA-Seq to analyze gene expression in our $T g$ (myo6b:RiboTag) model. We show that immunoprecipitated RNA from our $\mathrm{Tg}$ (myo6b:RiboTag) model is significantly enriched for known HC expressed transcripts, indicating that this model is effective in enriching for the HC translatome. Additionally, a comparison of our translatome dataset with a previously published zebrafish HC transcriptome dataset (generated using sorted HCs) shows that similar gene expression results can be obtained using the $\mathrm{Tg}$ (myo6b:RiboTag) model without cell sorting. Finally, we use the $\operatorname{Tg}$ (myo6b:RiboTag) model to identify novel HC expressed transcripts, and demonstrate that RiboTag immunoprecipitation helps to avoid gene expression changes that are induced by dissociation. Overall, we believe that this model will be highly useful for studying the normal development and function of 
zebrafish HCs, as well as changes to HC gene expression in response to different conditions.

\section{MATERIALS AND METHODS}

\section{Zebrafish Husbandry}

Zebrafish were grown at $28^{\circ} \mathrm{C}$ in E3 embryo media $(5 \mathrm{mM}$ $\mathrm{NaCl}, 0.17 \mathrm{mM} \mathrm{KCl}, 0.33 \mathrm{mM} \mathrm{CaCl}_{2}$, and $0.33 \mathrm{mM} \mathrm{MgSO}_{4}$ ) using standard methods. Work performed at the National Institute of Health was approved by the NIH Animal Use Committee under animal study protocol \#1362-13. At the University of Maryland School of Medicine (UMSOM), all procedures involving animals were carried out in accordance with the NIH Guide for the Care and Use of Laboratory Animals and have been approved by the Institutional Animal Care and Use Committee at the University of Maryland, Baltimore (protocol numbers 0514001 and 1116003).

\section{Vector Construction and Generation of the Tg(myo6b:GFP-2A-rp/10a-3xHA) Zebrafish}

Plasmid construction was based on the Tol2/Gateway zebrafish kit (Kwan et al., 2007). The pME-GFP-2A-rpl10a-3xHA was a generous gift from Dr. Brant Weinstein at the NIH. The p5Epmyo6b entry clone used to drive expression in hair cells has been described previously (Kindt et al., 2012). These two clones were used along with the tol2 kit gateway clones p3E-polyA (\#302) and pDest (\#394) to create the myo6b:GFP-2A-rpl10a$3 x H A$ expression construct. To generate a stable transgenic fish line, plasmid DNA at $50 \mathrm{ng} / \mu \mathrm{L}$ and tol 2 transposase mRNA at $20 \mathrm{ng} / \mu \mathrm{L}$ were injected into zebrafish embryos as previously described to create $\operatorname{Tg}$ (myo6b:GFP-2A-rpl10a-3xHA) ${ }^{\text {idc10 }}$ (Kwan et al., 2007). Each line was grown to the $F 1$ generation and outcrossed to confirm single copy integration.

\section{Immunohistochemistry and Confocal Imaging}

Immunohistochemistry was performed on whole-mount larvae. Wildtype or transgenic larvae were fixed with $4 \%$ paraformaldehyde in phosphate buffered saline (PBS) for $4 \mathrm{~h}$ at $4^{\circ} \mathrm{C}$. After $5 \times 5 \mathrm{~min}$ washes in PBS, followed by a $5 \mathrm{~min}$ wash in $\mathrm{H}_{2} \mathrm{O}$, larvae were permeabilized with ice cold acetone (at $-20^{\circ} \mathrm{C}$ ) for 5 min. Larvae were then washed in $\mathrm{H}_{2} \mathrm{O}$ for $5 \mathrm{~min}$, followed by a $5 \times 5 \mathrm{~min}$ washes in PBS, and then blocked overnight with PBS containing $2 \%$ goat serum and $1 \%$ bovine serum albumin (BSA). A primary rat anti-HA antibody (Roche) was diluted at 1:750 in PBS containing 1\% BSA, and larvae were incubated in the solution for $4 \mathrm{~h}$ at room temperature. After $5 \times 5 \mathrm{~min}$ washes in PBS to remove the primary antibody, an Alexa 568 secondary antibody diluted at 1:1,000 (Life Technologies) was added in PBS containing $1 \%$ BSA and incubated overnight at $4^{\circ} \mathrm{C}$. After $5 \times 5$ min washes in PBS to remove the secondary antibody, larvae were rinsed in $\mathrm{H}_{2} \mathrm{O}$ and mounted in Prolong Gold (Life Technologies). Fixed samples were imaged on an inverted Zeiss LSM 780 laser-scanning confocal microscope with a $63 \times 1.4 \mathrm{NA}$ oil objective lens. Excitation wavelengths of 488 and $546 \mathrm{~nm}$ were used to excite GFP and Alexa 568, respectively.

\section{Tg(myo6b:RiboTag) Translatome Immunoprecipitation}

The $T g$ (myo6b:RiboTag) immunoprecipitation protocol was modified from the RiboTag immunoprecipitation protocol described in Sanz et al. (2009). Briefly, GFP ${ }^{+} \operatorname{Tg}$ (myo6b:RiboTag) zebrafish larvae were euthanized at 5 days post fertilization (dpf) using MS-222/Tricaine and rinsed with system water. Groups of fifty larvae were then either flash frozen and stored at $-80^{\circ} \mathrm{C}$ or used immediately for immunoprecipitation. Larvae were resuspended and homogenized in $1 \mathrm{~mL}$ of supplemented homogenization buffer $(50 \mathrm{mM}$ Tris- $\mathrm{HCl}$ pH.7, $100 \mathrm{mM} \mathrm{KCl}, 12 \mathrm{mM} \mathrm{MgCl} 2,1 \%$ Nonidet P-40, $1 \mathrm{mM} \mathrm{1,4-}$ Dithiothreitol, $1 \mathrm{X}$ protease inhibitor cocktail, $200 \mathrm{U} / \mathrm{mL}$ RNAsin, $100 \mu \mathrm{g} / \mathrm{mL}$ cycloheximide, $1 \mathrm{mg} / \mathrm{mL}$ heparin) by douncing on ice. Homogenates were spun at $10,000 \mathrm{~g}$ for $10 \mathrm{~min}$ at $4^{\circ} \mathrm{C}$ to remove particulates, and a small sample of clear supernatant was reserved for total RNA isolation (input control, IN). Remaining supernatant was incubated with $5 \mu \mathrm{g}$ HA antibody (BioLegend) at $4^{\circ} \mathrm{C}$ under gentle rotation for $4-6 \mathrm{~h}$. After incubation, supernatants were incubated with $300 \mu \mathrm{L}$ of rinsed Invitrogen Dynabeads Protein $G$ magnetic beads (Thermo Fisher) overnight, rotating. Following incubation, bound beads were rinsed three times with $800 \mu \mathrm{L}$ high salt buffer $(50 \mathrm{mM}$ Tris- $\mathrm{HCl} \mathrm{pH} .7$, $300 \mathrm{mM} \mathrm{KCl}, 12 \mathrm{mM} \mathrm{MgCl} 2,1 \%$ Nonidet P- $40,1 \mathrm{mM} \mathrm{1,4-}$ Dithiothreitol, $100 \mu \mathrm{g} / \mathrm{mL}$ cycloheximide) at $4^{\circ} \mathrm{C}$ for $10 \mathrm{~min}$, rotating. After washing, $350 \mu \mathrm{L}$ of buffer RLT from the RNeasy Plus Micro kit (Qiagen) was added to the bound beads or reserved input sample and vortexed for $30 \mathrm{~s}$ to dissociate bound RNA. RNA was then extracted according to manufacturer's instructions (using $16 \mu \mathrm{L}$ of nuclease free water for elution) and stored at $-80^{\circ} \mathrm{C}$. RNA quality and concentration was assessed using the Agilent RNA Pico kit (Agilent Technologies) at the UMSOM Genomics Core Facility.

\section{Fluorescence Activated Cell Sorting}

Zebrafish dissociation and FACS were performed as previously described (Elkon et al., 2015). Briefly, approximately 150 $\mathrm{Tg}$ (myo6b:RiboTag) larvae per replicate were euthanized at $5 \mathrm{dpf}$ and dissociated in cold trypsin-EDTA solution $(0.5 \mathrm{~g} / \mathrm{L}$ trypsin, $0.2 \mathrm{~g} / \mathrm{L}$ EDTA, Sigma) by trituration with a p1000 pipette tip on ice for $20 \mathrm{~min}$. Dissociation was then halted by adding HBSS supplemented with $10 \%$ fetal bovine serum (FBS) and $100 \mu \mathrm{g} / \mathrm{mL}$ DNaseI. Cells were filtered through a $70 \mu \mathrm{m}$ cell strainer (Fischer Scientific) and pelleted by centrifugation at 2,000 rpm for $10 \mathrm{~min}$ at $4^{\circ} \mathrm{C}$. Cells were washed once in HBSS, resuspended in HBSS supplemented with $10 \% \mathrm{FBS}$, and filtered into glass tubes through a $35 \mu \mathrm{m}$ cell strainer (Falcon). Flow cytometry analyses were performed at the University of Maryland Marlene and Stewart Greenebaum Comprehensive Cancer Center Flow Cytometry Shared Service. Samples of GFP positive and negative cells (HCs and the rest of the fish) were collected using a BD FACSAria II (BD Biosciences), and a small aliquot of each sorted population was re-analyzed to determine cell purity. RNA was extracted from sorted cells using Trizol LS Reagent (Thermo Fischer Scientific) and the Direct-zol ${ }^{\mathrm{TM}}$ RNA MiniPrep Plus (Zymo Research). 


\section{RT-qPCR}

RT-qPCR was performed as described previously with minor modifications (Matern et al., 2017). RNA from $5 \mathrm{dpf}$ Tg(myo6b:RiboTag) zebrafish input and IP samples, or sorted cells, was reverse-transcribed using the Maxima First Strand cDNA Synthesis Kit (Thermo Fisher Scientific), and qPCR was performed using the Maxima SYBR Green/ROX qPCR Master Mix (Thermo Fisher Scientific). To account for low levels of RNA obtained from sorted cells, a preamplification step using PerfeCTa PreAmp Supermix (Quantbio) was added for the RNASeq validation and immediate early gene expression experiments. Expression values were normalized to $a c t b 1$ expression (see Supplementary Table 4 for primer sequences).

\section{RNA Sequencing and Informatics}

RNA from 5 dpf $T g$ (myo6b:RiboTag) zebrafish IN and IP samples was submitted in biological quadruplicates for RNA-Seq at the UMSOM Institute for Genome Sciences. Only samples with RNA integrity numbers $(\mathrm{RIN})>8$ were used for sequencing. Libraries were prepared from $25 \mathrm{ng}$ of RNA using the TruSeq RNA Sample Prep kit (Illumina) per manufacturer's instructions, with the exception of an additional PCR cycle. Samples were sequenced on an Illumina HiSeq 4000 with a 75 bp paired-end read configuration. Between 100 and 150 million reads were obtained for each sample, and reads were aligned to the zebrafish genome (Danio rerio.GRCz10) using TopHat version 2.0.8 (maximum number of mismatches $=2$; segment length $=30$; maximum multi-hits per read $=25$; maximum intron length $=50,000)(\mathrm{Kim}$ et al., 2013). The number of reads that aligned to the predicted coding regions were determined using HTSeq (Anders et al., 2015), and only genes with CPM (reads count per transcripts per million mapped reads) values $>0.01$ in all IN and IP replicates were called as expressed (17,164 genes). One IP sample had a high intergenic content suggestive of DNA contamination and was excluded from the analysis. See Supplementary Table 1 for alignment statistics. Significant differential expression was assessed using DEseq (Anders and Huber, 2010). RNASeq data were submitted to the Gene Expression Omnibus database (GEO accession GSE102861), as well as the gEAR Portal (UMgEAR.org). Gene ontology of highly enriched and depleted gene sets was performed using the Gene Ontology (GO) database (http://www.geneontology.org) (Harris et al., 2004). For $\mathrm{HC}$ enriched and depleted gene sets, top branches of GO terms are shown. Anatomical structure enrichment was performed using the Zebrafish Expression Ontology of Gene Sets (ZEOGS) tool (Prykhozhij et al., 2013), with a corrected $p$-value cutoff of 0.10 . Sorted HC dataset transcript IDs from Steiner et al. were converted to gene IDs using bioDBnet (https://biodbnet-abcc. ncifcrf.gov) (Mudunuri et al., 2009).

\section{RESULTS}

\section{Generation of Tg(myo6b:GFP-2A-rpl10a-3xHA) Zebrafish to Create the Tg(myo6b:RiboTag) Model}

To create a RiboTag zebrafish model that would allow for enrichment of the inner ear and lateral line HC translatome, we utilized the rpl10a gene, which has previously been used to effectively immunoprecipitate ribosomes in zebrafish via a GFP tag (Tryon et al., 2013). However, instead of an Rpl10aGFP fusion protein, here we use an Rpl10a-3xHA fusion protein for the ribosomal pulldown. To drive Rpl10a-3xHA expression in HCs we utilized the HC-promoter myo6b, which has been shown to specifically drive expression of downstream genes in zebrafish inner ear and lateral line HCs (Obholzer et al., 2008). The construct is engineered to express Rpl10a3xHA together with GFP bi-cistronically using the viral P2A peptide, resulting in the myo6b:GFP-2A-rpl10a-3xHA construct (Figure 1A). This construct was injected into zebrafish embryos at the one-cell stage to create a stable $\mathrm{Tg}$ (myo6b:GFP-2A-rpl10a$3 x \mathrm{HA}$ ) transgenic line, referred to here as our $\mathrm{Tg}$ (myo6b:RiboTag) model. To confirm Rpl10a-3xHA was localizing properly in HCs, we immunostained our $T g$ (myo6b:RiboTag) zebrafish with an anti-HA antibody at 5 or $6 \mathrm{dpf}$, when $\mathrm{HCs}$ in the inner ear and along the lateral line have developed. As expected, we observed both GFP and HA signal localizing to the cytosol of inner ear and lateral line HCs (Figures 1B,C). Consistent with previously observed Rpl10a-GFP localization in $x e f 1 \alpha>$ TRAP zebrafish (Tryon et al., 2013), the HA staining in the HCs of the Tg(myo6b:RiboTag) zebrafish also shows nucleolar localization (Figure 1C, white arrows), indicating that our model is localizing Rpl10a-3xHA properly.

\section{Ribosome Immunoprecipitation Isolates the HC Translatome From Tg(myo6b:RiboTag) Zebrafish}

In order to test whether the $T g$ (myo6b:RiboTag) zebrafish could be used to analyze gene expression of the inner ear and lateral line HCs, we next adapted the RiboTag immunoprecipitation protocol described in Sanz et al. (2009) to capture the HC translatome at $5 \mathrm{dpf}$ (Figure 2A) using fresh or frozen embryos. We chose $5 \mathrm{dpf}$ as the time point for our experiment, as this time point allows us to compare our results to other previously published HC gene expression datasets also generated using 5 dpf larvae. This technique yields $\sim 50 \mathrm{ng}$ of immunoprecipitated RNA (IP, average RNA concentration $=3.5 \pm 2.3 \mathrm{ng} / \mu \mathrm{L}$ in $16 \mu \mathrm{L}$ elution volume) and $660 \mathrm{ng}$ of input control RNA (IN, average RNA concentration $=41.3 \pm 24.6 \mathrm{ng} / \mu \mathrm{L}$ in $16 \mu \mathrm{L}$ elution volume) per fifty homogenized $\mathrm{Tg}$ (myo6b:RiboTag) larvae ( $n=19$ IPs). Because of the amount of homogenate used for immunoprecipitation, these quantities of RNA correspond to $\sim 330$ ng of input RNA and $\sim 1$ ng immunoprecipitated RNA per larvae. Interestingly, while analysis of the IN samples showed characteristic ratio of $1.6-2$ between the $28 \mathrm{~s}$ to $18 \mathrm{~s}$ rRNA, representing intact RNA components of the $60 \mathrm{~S}$ and $40 \mathrm{~S}$ ribosomal subunits respectively, the IP samples showed ratios $>2.5$, indicating reduced levels of 18s rRNA (Figure 2B). This observation is consistent with the zebrafish ribosomal immunoprecipitation results in Tryon et al. (2013), and is thought to be a result of tagging the $60 \mathrm{~S}$ subunit of the ribosome (of which Rpl10a is a component). Overall, these results indicate that high quality RNA in amounts suitable for downstream analyses such as RT-qPCR and RNA sequencing (RNA-Seq) can 
A

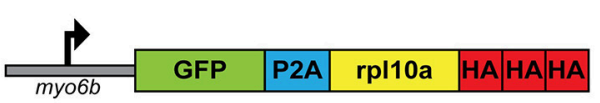

B

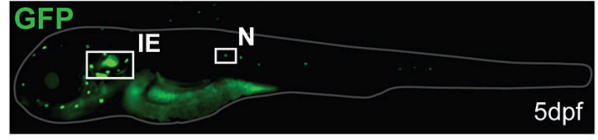

C

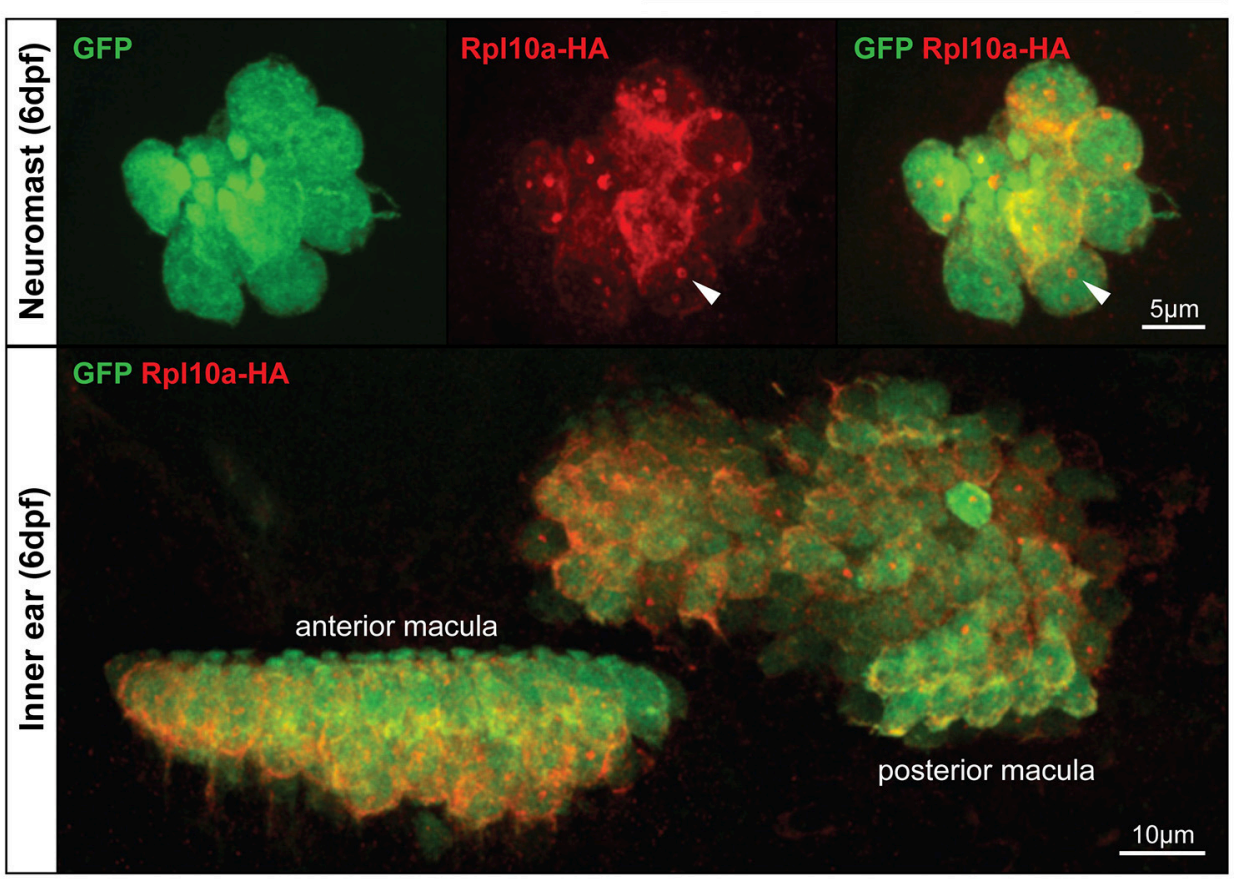

FIGURE 1 | The Tg(myo6b:RiboTag) zebrafish. (A) Schematic representation of the GFP-2A-rpl10a-3xHA construct driven by the HC-specific myo6b promoter. (B) Representative image of a live Tg(myo6b:RiboTag) zebrafish at $5 \mathrm{dpf}$ showing GFP expression in the inner ear (IE) and lateral line neuromasts (N).

(C) Immunohistochemistry using an HA antibody showing that Rpl10a-HA expression overlaps with GFP expression specifically within the HCs of the inner ear and neuromasts. White arrows denote nucleolar Rpl10a-HA staining.

be obtained using the $T g$ (myo6b:RiboTag) fish along with our immunoprecipitation protocol.

After establishing our protocol, we next aimed to validate whether the immunoprecipitation step was efficiently pulling down RNA from HCs. For this analysis, we used RT-qPCR to determine the relative transcript abundance of known $\mathrm{HC}$ expressed genes between IN and IP samples. As expected, immunoprecipitation of HA-tagged ribosomes resulted in significant enrichment of transcripts for the HC expressed genes atoh $1 a$, which encodes a transcription factor necessary for HC development, and myo6b, the promoter of which is used to drive rpl10a-3xHA expression in this model (Figure 2C) (Seiler et al., 2004; Millimaki et al., 2007). We did not observe significant differences in transcript abundance for the supporting cell expressed gene sox 2 or the muscle expressed gene myod1. However, we observed significant depletion of the eye specific gene rho and gut specific gene and vil1 in the RNA obtained from the IP compared to the IN. These results indicate that the protocol adapted for the $T g$ (myo6b:RiboTag) ribosome immunoprecipitation is able to specifically enrich for transcripts of HC expressed genes, while also depleting, to a varying extent, transcripts expressed in other cell types.

\section{RNA Sequencing of IP and in Samples From Tg(myo6b:RiboTag) Zebrafish}

After confirming the efficiency of immunoprecipitation protocol, we next sought to perform an unbiased and systematic analysis of the utility of the $\mathrm{Tg}$ (myo6b:RiboTag) model as a tool to study the zebrafish HC translatome. We therefore performed RNASeq on IP and IN samples in atleast three biological replicates from $5 \mathrm{dpf} T g$ (myo6b:RiboTag) zebrafish. In total, 17,164 genes were detected as expressed in our IN and IP samples based on our criteria (IN and IP counts per million [CPM] $>0.01$ in all samples), allowing for a direct comparison of expression levels (see Supplementary Data Sheet 1). Of these, transcripts for 2,379 genes were significantly enriched in IP samples compared to IN (fold change $>2$, IP CPM $>1$, false discovery rate $[$ FDR] $<0.05$ ), and transcripts of 2,258 genes were significantly depleted in IP samples compared to IN (fold change $<0.5$, IN CPM $>1$, FDR $<0.05$ ) (Figure 3A). As an internal control, we first analyzed transcript enrichment and depletion of the same HC and non$\mathrm{HC}$ expressed genes used in our RT-qPCR analysis (Figure 2C) and saw parallel results (Figure 3B). Our RNA-Seq analysis also found that transcripts for the known HC genes atoh1 $a$ and myo6b are significantly enriched in the IP samples compared to IN. 


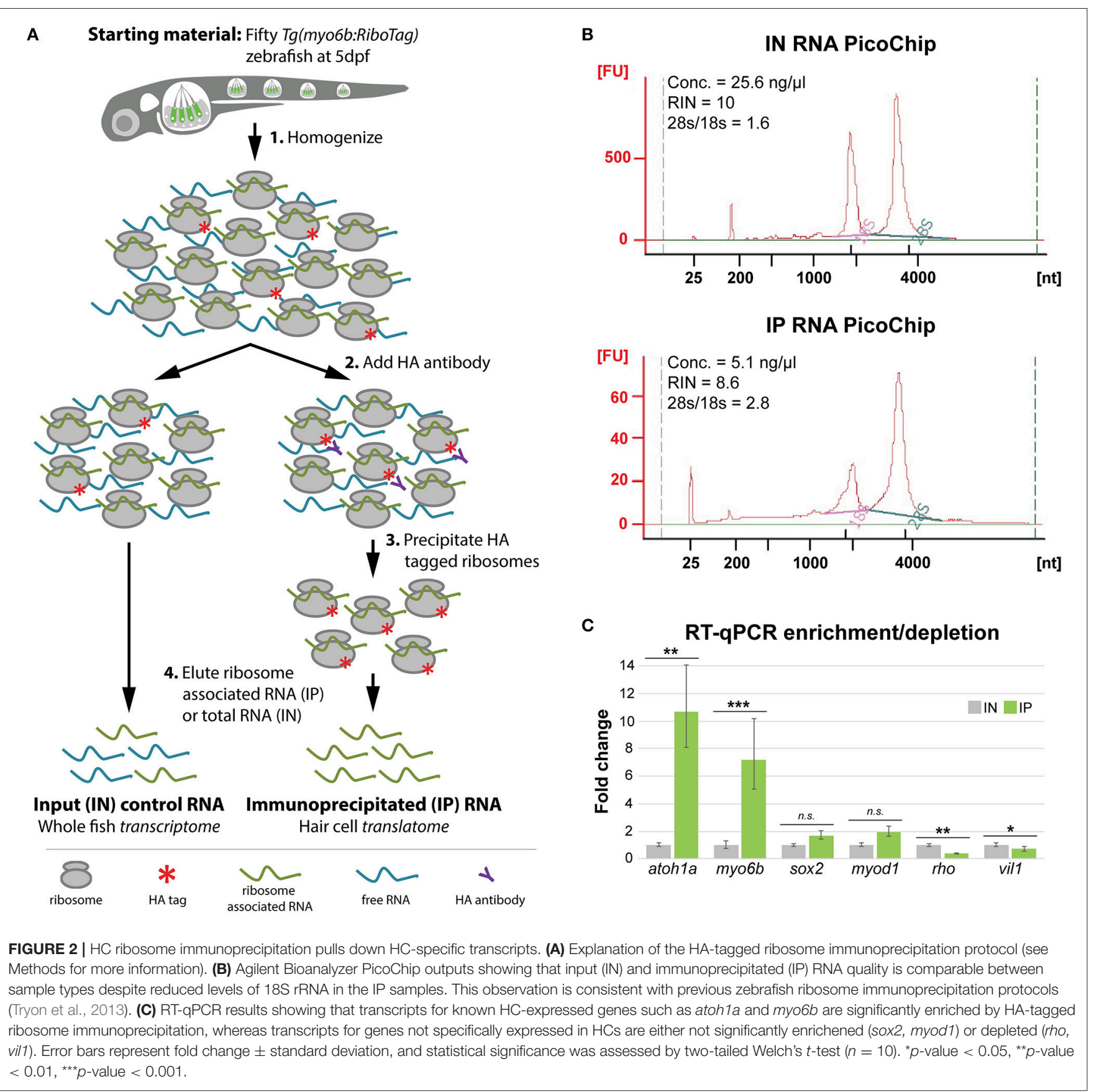

As shown in Figure 3A, the majority of transcripts found to be significantly enriched or depleted in the IP compared to the IN samples had a two to five-fold-change in transcript abundance. This large number of genes with small differences in transcript abundance between IP and IN could be attributed to the likelihood that many HC-expressed genes are also expressed in other cell types. Because our protocol isolates RNA from whole, intact larvae, concomitant enrichment and depletion of RNA expressed in both HCs and other cell types could lower the overall fold change values for $\mathrm{HC}$ enrichment and depletion. Therefore, in order to restrict our analyses to genes with a preferential expression in HCs compared to other tissues, we focused on genes with high fold-change transcript enrichment in the IP samples. A gene ontology (GO) analysis of the genes with five-fold or greater transcript enrichment in IP compared to IN samples $(n=694)$ identified "detection of mechanical stimulus" as a top enriched GO term (Table 1, GO associated with $\mathrm{HC}$ function in bold). This same analysis was also performed on the genes with five-fold or greater depletion in the IP compared to IN samples $(n=532)$, resulting in top GO terms including "detection of light stimulus" and "visual perception" (Table 2, in bold). Together with our RT-qPCR, our RNA-Seq, 


\section{A Distribution of significantly enriched and depleted transcripts}

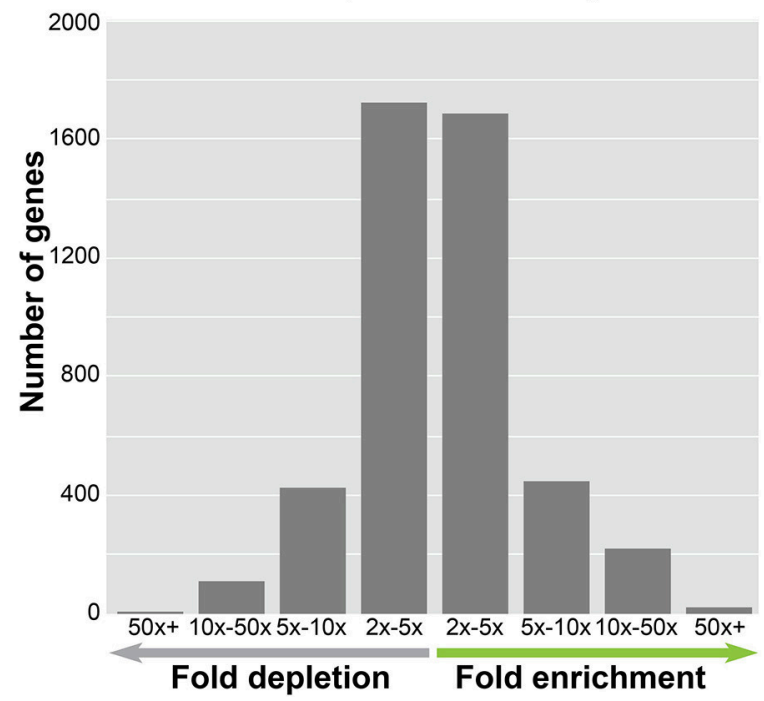

B

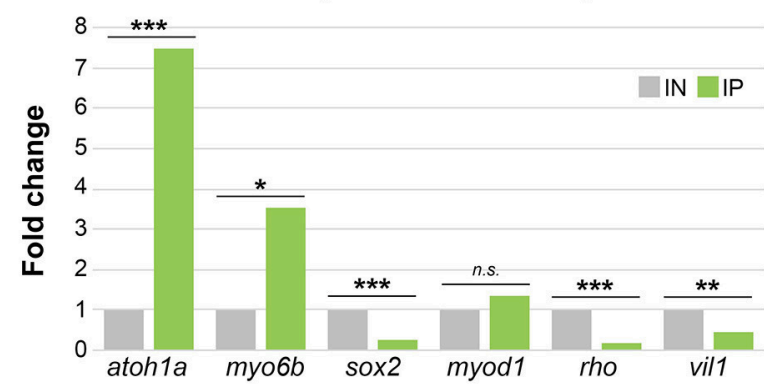

FIGURE 3 | HC ribosome immunoprecipitation can reliably detect $\mathrm{HC}$ enriched and depleted transcripts by RNA-Seq. (A) Bar graph showing the distribution of significantly enriched and depleted transcripts in the IN vs. IP samples binned by fold change range. For genes with depleted transcripts in the IP samples, the number of genes per bin is as follows: $2 x-5 x=1726,5 x-10 x$ $=422,5 x-10 x=109$, and 50x $+=1$. For IP enriched gene transcripts, the number of genes per bin is as follows: $2 x-5 x=1685,5 x-10 x=450,5 x-$ $10 x=223$, and $50 x+=21$. (B) RNA-Seq fold change enrichment and depletion of HC expressed and non-expressed genes replicates the results obtained by RT-qPCR (Figure $\mathbf{2 C}$ ). Statistical significance was assessed using DEseq (see Methods). ${ }^{\star} \mathrm{FDR}<0.05,{ }^{\star \star} \mathrm{FDR}<0.01,{ }^{\star \star \star} \mathrm{FDR}<0.001$.

and GO analysis of five-fold enriched transcripts support that our $\operatorname{Tg}$ (myo6b:RiboTag) model and immunoprecipitation protocol can enrich for the zebrafish HC translatome.

\section{Immunoprecipitation Enriches for Known HC Expressed Transcripts}

We next wanted to assess the efficiency of our model for enriching for other genes known to be expressed in zebrafish HCs. Therefore, we analyzed our transcripts with five-fold or greater enrichment $(n=694)$ and depletion $(n=532)$, from here on name "enriched" and "depleted" gene sets, using the Zebrafish Expression Ontology of Gene Sets (ZEOGS) tool (Prykhozhij et al., 2013). This online tool can be used to detect overrepresented anatomical structures based on known gene expression patterns from the Zebrafish Information Network (ZFIN) (Howe et al., 2013). When analyzing the enriched gene set, the top result for overrepresented anatomy is "neuromast," and also includes the inner ear HC types "hair cell anterior macula" and "hair cell posterior macula" (Table 3). We also performed a ZEOGS analysis on the highly depleted genes, resulting in the top term of "retinal photoreceptor layer" and no results related to the inner ear or neuromast hair cells (Supplementary Table 2). This indicates that the $T g$ (myo6b:RiboTag) immunoprecipitation is capturing transcripts of genes experimentally validated to be expressed in neuromast and inner ear HCs.

Zebrafish have proved to be a highly useful animal model for studying the normal development and function of HCs, as well as for dissecting the mechanistic consequences of gene mutations that cause human hearing loss (Lieschke and Currie, 2007). For this reason, we next probed our RNA-Seq dataset to detect zebrafish homologs of known human syndromic and non-syndromic deafness causing genes reported on the Hereditary Hearing Loss Homepage (http:// hereditaryhearingloss.org) (Van Camp and Smith, 2017). In total, we found that 36 deafness genes were significantly enriched in our zebrafish HC IP samples compared to IN (fold change $>2, p$-value $<0.05$ ), of which 27 remained significant after taking into account multiple testing (Table 4, bold values denote FDR < 0.05). Importantly, only 13 of these genes have been previously reported in the literature to be expressed in the developing zebrafish inner ear or HCs, which indicates that the $\mathrm{Tg}$ (myo6b:RiboTag) model is effective in identifying genes not previously known to be expressed in zebrafish HCs.

\section{Tg(myo6b:RiboTag) Gene Expression Profile Is Comparable to Previously} Published FACS-Based HC Transcriptomes

In order to ensure that similar HC gene enrichment could be obtained from our $\mathrm{Tg}$ (myo6b:RiboTag) immunoprecipitation method, we next wanted to compare our translatome dataset to a previously published cell sorting experiment. To do this, we utilized a dataset generated by comparing gene expression in sorted zebrafish HCs to sorted skin cells using microarray technology (Steiner et al., 2014). Setting a five-fold transcript enrichment criterion in HCs compared to skin cells within the microarray dataset, we identified 1,041 unique gene IDs with significant enrichment in sorted HCs $(p$-value $<0.05$ from Steiner et al., 2014 results). We then analyzed the fold change values of this set of genes within our $\mathrm{Tg}$ (myo6b:RiboTag) dataset. Of the 1,041 genes identified as significantly enriched in the Steiner et al. dataset, 762 were identified as expressed in our dataset and could be used in this analysis. This is secondary to our strict CPM cutoff to determine gene expression (CPM > 0.01 for all IP and IN samples), as all 1,041 genes are found within our dataset before CPM filtering. Similar to the Steiner et al. dataset, the transcripts encoding these 762 genes also had 
TABLE 1 | Gene ontology analysis of hair cell enriched genes.

\begin{tabular}{|c|c|c|c|c|c|c|}
\hline GO biological process complete & \# in Reference & \# in input & \# expected & $\begin{array}{l}\text { Fold } \\
\text { enrichment }\end{array}$ & FDR & Genes \\
\hline $\begin{array}{l}\text { miRNA mediated inhibition of } \\
\text { translation }\end{array}$ & 9 & 5 & 0.21 & 24.32 & 0.00252 & trim71 tnrc6b tnrc6a tnrc6c1 ago2 \\
\hline Skeletal muscle contraction & 23 & 5 & 0.53 & 9.52 & 0.0373 & CU929259 tnni1c tnnc1b CU929259 tnni1d \\
\hline $\begin{array}{l}\text { detection of mechanical } \\
\text { stimulus }\end{array}$ & 25 & 5 & 0.57 & 8.76 & 0.0496 & tmc2a loxhd1a loxhd1b dennd5a Ihfp/5a \\
\hline $\begin{array}{l}\text { sensory perception of } \\
\text { mechanical stimulus }\end{array}$ & 48 & 7 & 1.1 & 6.37 & 0.0283 & tmc2a pcdh15b atp2b1a Ihfpl5a mecp2 BX572619 dcdc2b \\
\hline neuromast development & 62 & 8 & 1.42 & 5.64 & 0.0237 & $\begin{array}{l}\text { atoh1a pcsk5a slc12a5b atp2b1a pho BX572619 erbb2 } \\
\text { dcdc2b }\end{array}$ \\
\hline $\begin{array}{l}\text { Steroid hormone mediated } \\
\text { signaling pathway }\end{array}$ & 77 & 9 & 1.76 & 5.11 & 0.0198 & vdra abhd2a pparda thrab rorb rorcb nr6a1b thrb nrob2a \\
\hline mRNA metabolic process & 299 & 19 & 6.84 & 2.78 & 0.0182 & $\begin{array}{l}\text { celf4 ptbp3 qkib rbbp6 rbmx2 crnkl1 snrnp70 tnrc6a nova2 } \\
\text { rbm25a exosc7 qkia kiaa0907 exosc3 coil snrpa aqr dicer1 } \\
\text { ago2 }\end{array}$ \\
\hline Transcription, DNA-templated & 841 & 43 & 19.24 & 2.23 & 0.00093 & $\begin{array}{l}\text { hdac4 hoxb1a pou6f2 vdra nfia rest stat2 hoxb3a foxh1 brf2 } \\
\text { pparda hoxc6b bhlhe41 hoxa1a thrab rorb ncoa1 gtf2a1l } \\
\text { nfat5a eed rorcb mef2b nrarpa mef2aa nr6a1b clocka } \\
\text { med19a pou2f1b tcf3a jarid2b thrb onecut2 BX005395 } \\
\text { twistnb onecut1 mecp2 ccnd1 hoxa1a znf367 nr0b2a } \\
\text { pou2f2a asxl2 nfic }\end{array}$ \\
\hline $\begin{array}{l}\text { Regulation of transcription, } \\
\text { DNA-templated }\end{array}$ & 1703 & 71 & 38.97 & 1.82 & 0.00092 & $\begin{array}{l}\text { hdac4 hoxb1a nkap pou6f2 pde8b vdra nfia rest mkl2a stat2 } \\
\text { hoxb3a foxh1 brf2 pparda hoxc6b has2 tcf3a bhlhe41 } \\
\text { hoxa1a thrab gfi1aa trps1 rorb atoh1a foxj3 ncoa1 crebrf } \\
\text { zfhx4 fos/1a nfat5a eed ifx7 rorcb tomm20a ches } 1 \text { mef2b } \\
\text { nrarpa CU633479 mef2aa nr6a1b clocka hmbox1b med19a } \\
\text { pou2f1b tcf3a jarid2b thrb BX511021 cica onecut2 pbxip1b } \\
\text { zfhx3 BX005395 onecut1 mecp2 rbpja foxb1b ccnd1 hnf1a } \\
\text { hoxa1a tb/1xr1b mycbp znf367 nr0b2a pou2f2a crtc1a ago2 } \\
\text { asxl2 nfic dot1l rbpjb }\end{array}$ \\
\hline
\end{tabular}

a higher enrichment in HCs according to our dataset (IP vs. IN, mean $\log _{2} \mathrm{FC}=0.51$ ), compared to all other transcripts detected as expressed (mean $\log _{2} \mathrm{FC}=0.085$ ) (Figure 4A). This result suggests that similar $\mathrm{HC}$ expression results can be obtained using either cell sorting or the $\mathrm{Tg}$ (myo6b:RiboTag) zebrafish.

A possible concern with dissociation and FACS-based approaches for analysis of gene expression relates to induction of changes in gene expression secondary to cellular trauma, loss of tissue context (e.g., cell-cell contacts and lateral inhibition), and length of time from dissociation to RNA extraction. Indeed, a recent manuscript published by van den Brink et al. described robust induction of immediate early gene expression (i.e., Fos, $J u n$, and Egr1), as well as heat shock protein genes, in mouse muscle stem cells that were dissociated for single cell RNA-Seq (van den Brink et al., 2017). We therefore wanted to compare the expression of the zebrafish homologs of selected immediate early and heat shock protein encoding genes between sorted HCs and immunoprecipitated $\mathrm{HC}$ transcripts in the $\mathrm{Tg}$ (myo6b:RiboTag) IP. For this analysis, we utilized the concomitant myo6b promoter driven expression of GFP in the HCs of the Tg(myo6b:RiboTag). Five-day-old larvae were dissociated and cells were separated by FACS to GFP-positive (HCs) and GFP-negative (cells from the rest of the fish) populations (Figure 4B). Using RT-qPCR, we found that the immediate early genes egrl, fosab, fosb, and junb, as well as the zebrafish heat shock protein gene $h s p 70.1$ were enriched in sorted HCs compared to their level of expression in intact fish (Figure 4C). Similarly, all five genes were highly expressed in the sorted GFP-negative cells in comparison to their expression in intact zebrafish (Figure 4D), suggesting that their expression is likely induced by the dissociation and cell sorting process. Finally, a similar RT-qPCR analysis performed on the $\operatorname{Tg}$ (myo6b:RiboTag) IP vs. IN samples showed that only two of these transcripts, egr 1 and fosb are enriched in the IP (Figure 4E). Taken together, these data suggest that (1) immediate early genes are induced in the sorted samples, and (2) that this technical artifact is avoided by measuring gene expression using the $\operatorname{Tg}$ (myo6b:RiboTag) zebrafish.

\section{Immunoprecipitation of the HC Translatome Reveals Novel HC Expressed Genes}

By comparing our data to genes previously identified as HC-specific in zebrafish, we have demonstrated that the $\operatorname{Tg}$ (myo6b:RiboTag) immunoprecipitation method allows for detection of HC expressed genes in zebrafish. However, many of the transcripts that were detected as highly enriched in the IP were not previously reported in the literature as expressed in zebrafish HCs. For this reason, we selected 10 genes that have not been previously characterized as expressed in zebrafish HCs from among our top 100 significantly enriched transcripts (see Supplementary Table 3) for validation of expression by RTqPCR. These genes were acin1a, cnga1, cnga3a, cnga3b, dynlrb2, grin $2 d b$, loxhd1a, loxhd1b, onecut1, and $z b t b 20$. First, we utilized independent $\mathrm{Tg}$ (myo6b:RiboTag) IP and IN samples, and found that eight out of the 10 genes validated as significantly enriched 
TABLE 2 | Gene ontology analysis of hair cell depleted genes.

\begin{tabular}{|c|c|c|c|c|c|c|}
\hline $\begin{array}{l}\text { GO biological process } \\
\text { complete }\end{array}$ & \# in Reference & \# in Input & \# expected & $\begin{array}{l}\text { Fold } \\
\text { enrichment }\end{array}$ & FDR & Genes \\
\hline $\begin{array}{l}\text { Antibiotic catabolic } \\
\text { process }\end{array}$ & 4 & 3 & 0.07 & 41.55 & 0.024 & esd cat amdhd2 \\
\hline $\begin{array}{l}\text { ATP synthesis coupled } \\
\text { electron transport }\end{array}$ & 43 & 7 & 0.78 & 9.02 & 0.00537 & $\begin{array}{l}\text { AC024175.9 (associated with } m t-n d 4 I m t-c y b m t-n d 2 m t-c o 1 \\
m t-n d 4 m t-n d 5)\end{array}$ \\
\hline $\begin{array}{l}\text { Detection of light } \\
\text { stimulus }\end{array}$ & 38 & 6 & 0.69 & 8.75 & 0.0176 & $\begin{array}{l}\text { opn1mw1 gnat2 rho lamc1 opn1sw1 (also associated with } \\
\text { opn1sw2) }\end{array}$ \\
\hline $\begin{array}{l}\text { Pyruvate metabolic } \\
\text { process }\end{array}$ & 43 & 6 & 0.78 & 7.73 & 0.0275 & hkdc1 aldoca eno2 pdha1b aldoaa pkmb \\
\hline ATP biosynthetic process & 64 & 8 & 1.16 & 6.93 & 0.00767 & $\begin{array}{l}\text { hkdc1 aldoca eno2 AC024175.9 (associated with mt-atp6) aldoaa } \\
\text { BX901937 pkmb }\end{array}$ \\
\hline Visual perception & 97 & 12 & 1.75 & 6.85 & 0.00022 & $\begin{array}{l}\text { opn1mw1 kera gnat2 rho lamc1 cryaa crx aoc2 irbp vsx2 } \\
\text { opn1sw1 (also associated with opn1sw2) }\end{array}$ \\
\hline $\begin{array}{l}\text { Proton transmembrane } \\
\text { transport }\end{array}$ & 57 & 7 & 1.03 & 6.8 & 0.0194 & $\begin{array}{l}\text { atpv0e2 AC024175.9 (associated with } m t-c 01 \text { mt-atp6) } \\
\text { BX901937 atp6v0b atp6v1b2 }\end{array}$ \\
\hline Regulation of cell growth & 97 & 9 & 1.75 & 5.14 & 0.0179 & $\begin{array}{l}\text { igfbp7 osgn1 ncaldb chrna1 casp9 epb41/3b lamtor2 dpys/2b } \\
\text { arl3/1 }\end{array}$ \\
\hline $\begin{array}{l}\text { Coenzyme biosynthetic } \\
\text { process }\end{array}$ & 116 & 9 & 2.1 & 4.3 & 0.0436 & aldoca eno2 spra mat2al pdha1b aldoaa ndufa9b pkmb \\
\hline $\begin{array}{l}\text { Nucleobase-containing } \\
\text { compound catabolic } \\
\text { process }\end{array}$ & 153 & 11 & 2.77 & 3.98 & 0.0239 & $\begin{array}{l}\text { hkdc1 aldoca eno2 upf3a smg5 pcid2 polr2gl aldoaa dis3 } \\
\text { dnase1/3l pkmb }\end{array}$ \\
\hline $\begin{array}{l}\text { Cellular protein } \\
\text { localization }\end{array}$ & 497 & 23 & 8.99 & 2.56 & 0.0115 & $\begin{array}{l}\text { vps11 copb1 ap2m1b ap4e1 smg5 tomm40l vps29 pcid2 glrbb } \\
\text { chrna1 atg9a copa wipi2 epb41/3b BX901937 grpel2 nmd3 } \\
\text { hsc70 sx1b napgb pttg1ipb sec24d lamtor2 }\end{array}$ \\
\hline Protein transport & 530 & 24 & 9.59 & 2.51 & 0.0113 & $\begin{array}{l}\text { arcn1b vps11 rab4a copb1 ap2m1b ap4e1 smg5 tomm40l vps29 } \\
\text { pcid2 rab10 tsg101a atg9a copa tvp23b BX901937 (associated } \\
\text { with Zgc:165520 vps37c) grpel2 nmd3 snx1b napgb jagn1a } \\
\text { pttg1ipb sec24d }\end{array}$ \\
\hline System development & 2,933 & 79 & 52.94 & 1.49 & 0.0437 & $\begin{array}{l}\text { tfap2b cdh7 tyrp1b inpp5b wif1 vps11 rab4a ecrg4b igfbp7 } \\
\text { copb1 slc2a2 slc35b2 col17a1b ponzr1 epb41b ncaldb fosab } \\
\text { rab10 slc4a1a PPP1CC pou4f2 slc33a1 dacha ruvbl2 chrna1 } \\
\text { snapc2 gdpd3a celsr2 copa rapsn ephb4a smyd1b casp9 lingo2a } \\
\text { inpp5kb mcm3 olfm2a aldoaa grhl2a rtn4r BX470189 fez1 lamc1 } \\
\text { dhps cryaa itm2bb id4 klhl40a camk1db crx Irfn4b rel Ihx1a jagn1a } \\
\text { inab scinla lamb2 padi2 anos1a slitrk6 zic6 CRIP2 plppr1 pttg1ipb } \\
\text { sec24d polr3b ugdh SLITRK1 atp6v0b dpysl2b mab21/2 pou3f1 } \\
\text { arl3/1 hpse Irrn1 PDCL3 dnase1/3l spry4 six3a }\end{array}$ \\
\hline
\end{tabular}

in IP compared to IN RNA samples (Figure 5A). To then further confirm the expression of these genes in HCs, we utilized RNA extracted from sorted HCs (post-sort purity $=95.3 \%$, see Figure 4C). All 8 genes that validated as enriched in our IP vs. IN samples were also detected as expressed in sorted zebrafish HCs (cycle threshold $[\mathrm{CT}]$ values $\leq 30$, Figure 5B). These data further show the utility of the $\operatorname{Tg}$ (myo6b:RiboTag) zebrafish in detecting $\mathrm{HC}$ expressed genes, as well as for the discovery of new genes with potential novel functions in HCs.

\section{DISCUSSION}

Cell type-specific expression analysis has gained popularity in the past decade, and is of particular importance to understanding the biology of rare cell types. The HCs of the zebrafish lateral line and inner ear are one such example of a rare cell type present within a complex tissue environment. The Tg(myo6b:RiboTag) zebrafish described here was generated to express both GFP and HA-tagged ribosomes in inner ear and lateral line HCs (Figures 1A-C). Thus, this model enables HC-specific gene expression analysis through two independent approaches: (1) tissue dissociation and cell
TABLE 3 | ZEOGS analysis of HC enriched transcripts.

\begin{tabular}{|c|c|c|}
\hline Anatomical term & Corrected $p$-value & Genes \\
\hline Neuromast & 0.00052 & $\begin{array}{l}\text { cabp2b morn3 pcsk5a rorb } \\
\text { s100t atp2b1a gfilaa otofa wasa } \\
\text { pho bdnf pvalb8 tmc2a tmc2b } \\
\text { s100s myclb atoh1a }\end{array}$ \\
\hline Levator operculi & 0.06849 & tnnc1b smyhc2 \\
\hline $\begin{array}{l}\text { Hair cell anterior } \\
\text { macula }\end{array}$ & 0.07032 & atp2b1a tmc2a otofa \\
\hline Hyohyoideus & 0.07515 & myha tnnc1b smyhc2 \\
\hline Olfactory epithelium & 0.0832 & $\begin{array}{l}\text { tnks } 1 \text { bp1 cnga3a s100t dlg2 } \\
\text { bdnf s100s s100a1 elav/3 }\end{array}$ \\
\hline Olfactory bulb & 0.0915 & $\begin{array}{l}\text { klf7a mef2aa fabp10b plxnb2b } \\
\text { s100t dlg2 bdnf pvalb8 s100s } \\
\text { cadm1b igdcc3 }\end{array}$ \\
\hline $\begin{array}{l}\text { Hair cell posterior } \\
\text { macula }\end{array}$ & 0.09839 & atp2b1a tmc2a \\
\hline
\end{tabular}

sorting based on GFP expression, and (2) immunoprecipitation of HA-tagged ribosomes to enrich for $\mathrm{HC}$-expressed transcripts. 
TABLE 4 | Fold change enrichment of human deafness gene homologs in Tg(myo6b:RiboTag) IP samples.

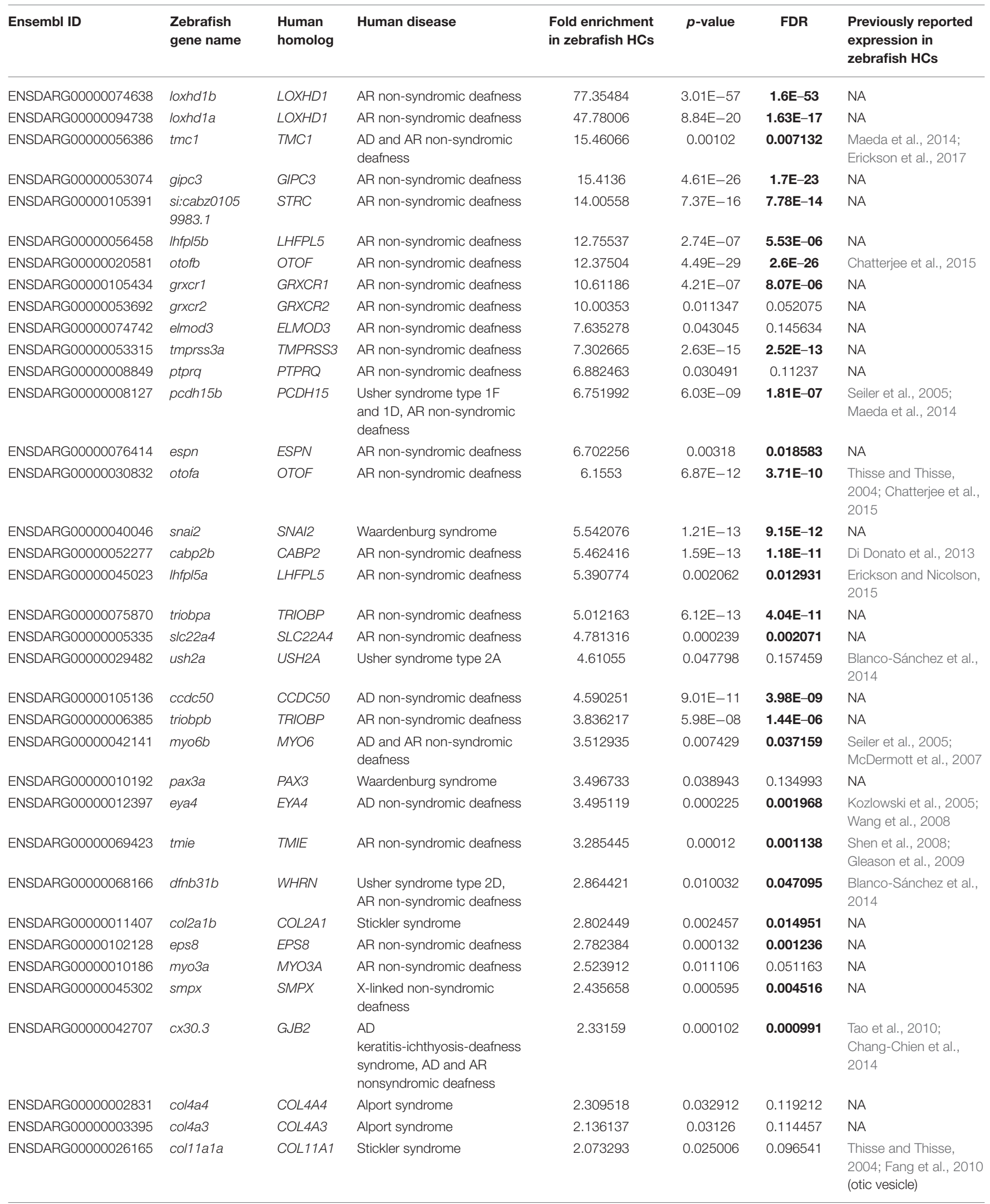

$A R$, autosomal recessive; $A D$, autosomal dominant. 


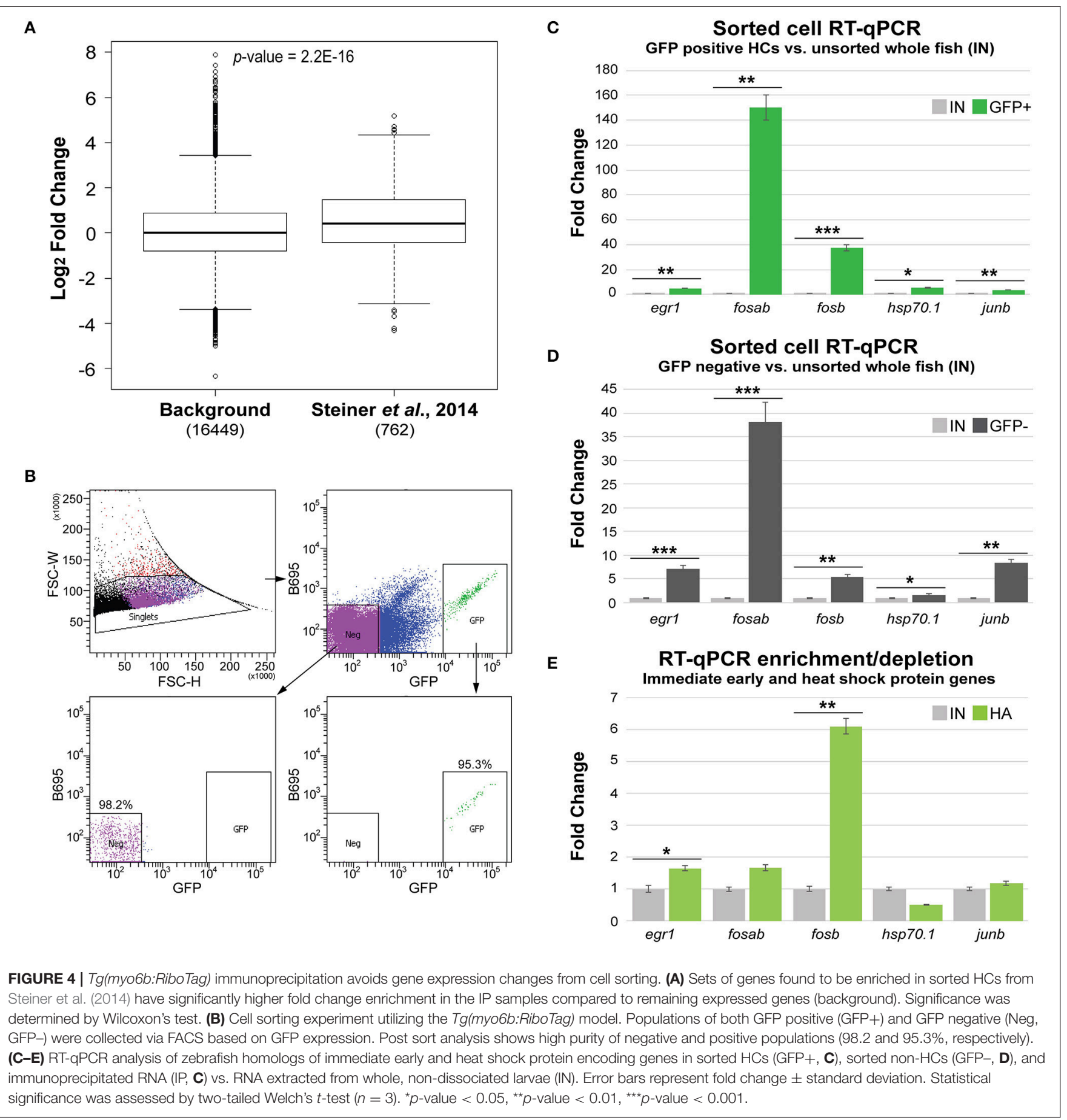

While HC-specific transcriptome analysis using flow cytometry is not novel, this is the first application of a RiboTag approach for immunoprecipitation of the zebrafish HC translatome, as well as the first model to allow HC-specific expression analysis using two separate approaches in zebrafish. Through the analyses presented in this manuscript, we have demonstrated the utility of the $\mathrm{Tg}$ (myo6b:RiboTag) model in enriching for HC-expressed transcripts (through the immunoprecipitation approach), while concurrently avoiding changes to gene expression that occur secondary to tissue dissociation and the cell sorting process. The immunoprecipitation protocol can be performed using either fresh or frozen tissues, making it a convenient technique for analysis of gene expression at multiple time points or treatment conditions, and removing the need for multiple cell sorting sessions. Our subsequent RNA-Seq and validation experiments revealed not only the expected enrichment of known zebrafish 
HC expressed transcripts, but also enrichment of transcripts with potential new functions in inner ear and/or lateral line HCs.

In light of these results, some important considerations should also be taken into account when using this model. First, unlike cell sorting where, in ideal conditions, the RNA will contain genetic material only from the cell types of interest, all ribosome immunoprecipitation models, including the $\operatorname{Tg}$ (myo6b:RiboTag) zebrafish, are not completely effective at isolating the translatome of a cell type of interest. Immunoprecipitated samples contain, to a varying extent, RNA from other cell types. Indeed, our average IP RNA yield ( $56 \mathrm{ng}$ per 50 larvae at $5 \mathrm{dpf}$ ) was much larger than would be expected if this technique was specifically acquiring only actively translated RNA from HCs.

Second, the inclusion of transcripts from other cell types limits the interpretation of the data. The HC translatome results described in this manuscript were generated by comparing immunoprecipitated HC RNA to the whole fish transcriptome, detecting 2,379 genes as more than two-fold enriched in HCs. This method of comparison is not ideal for detecting all HC expressed transcripts, as it is estimated that an average 11,00013,000 genes are expressed in all cell types (Ramsköld et al., 2009). Some transcripts which are expressed in HCs may not be detected as enriched if they are expressed in many other cell types included in the sample, as the effect of the depletion may (although not always), outweigh the effect of the enrichment. We therefore recommend that the $T g$ (myo6b:RiboTag) model be used to analyze gene expression in HCs between different conditions (e.g., drugs or noise) through direct comparison of the IP RNA. The IN RNA can be used to validate whether the changes in gene expression originate from HCs, and we recommend a two-fold enrichment cutoff in IP vs. IN to denote HC expression.

Third use of this model inherently relies on the expression of HA-tagged ribosomes in HCs under control of the myo6b promoter. This promoter is highly specific to HCs to all time points, which is critical to the successful use of this method and the purity of the immunoprecipitated RNA. However, analysis of the HC translatome in different conditions will therefore rely on (1) the presence of HCs and (2) the expression of myo6b. As examples, HC gene expression in very early developmental time points prior to the onset of myo6b, mutants in which HCs never develop or do not express myo6b, or treatments that rapidly decrease HC number or abolish myo6b expression, may be difficult or impossible to analyze with the $T g$ (myo6b:RiboTag) zebrafish.

Finally, the immunoprecipitation protocol is limited by the total amount of tissue that can be homogenized in one sample (we recommend not exceeding $10 \%$ the total volume of homogenization buffer). For analyses of the HC translatome at later developmental stages, different dissection techniques can be applied to reduce total tissue volume and "pre-enrich" for HCs (i.e., inner ear dissection, skin peeling, etc.). These dissections may also be applied to isolate and compare gene expression differences in specific HC subtypes (i.e., inner ear vs. neuromast). Overall, each of these points should be considered when designing experiments using this model.

Our results show that the $T g$ (myo6b:RiboTag) zebrafish immunoprecipitation protocol detailed in the methods yields quantities of RNA appropriate for downstream analyses of HC

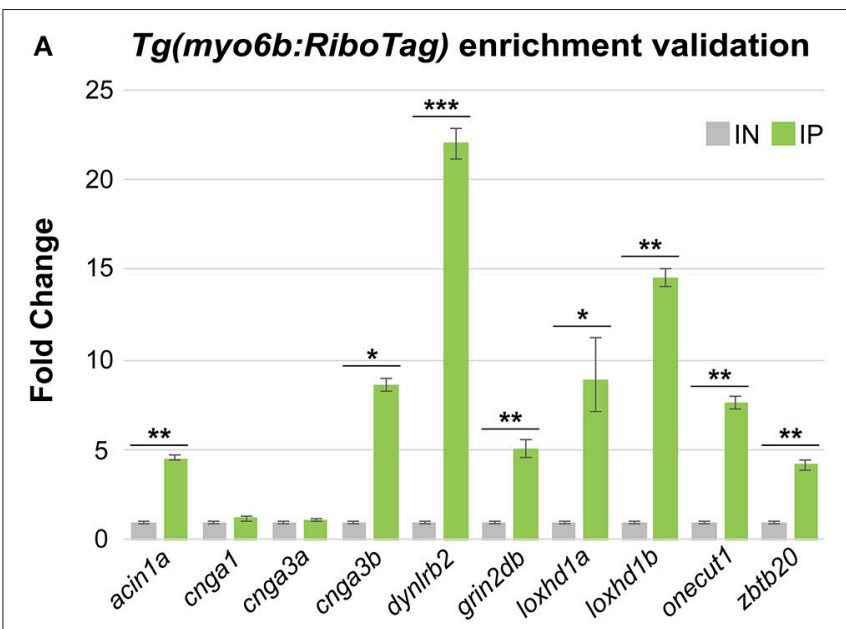

\section{B Sorted HC expression validation}

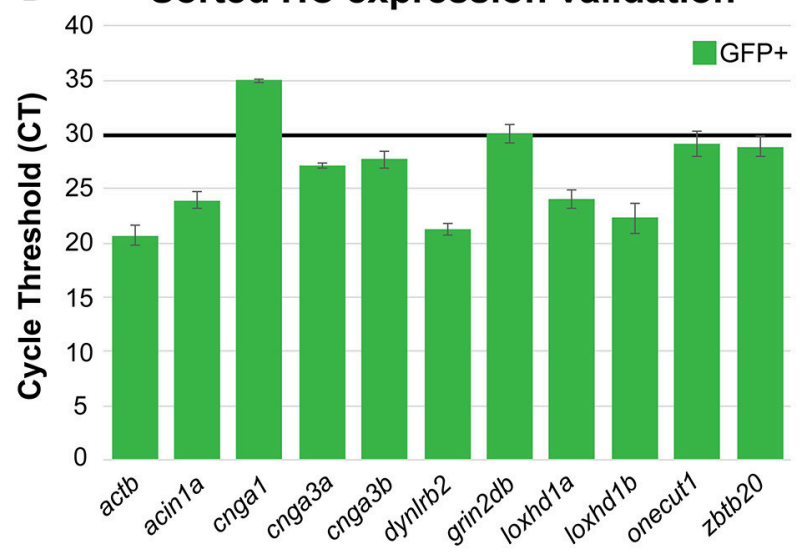

FIGURE 5 | HC RiboTag immunoprecipitation reveals expression of novel HC expressed genes. (A) Validation of 10 genes found to be highly enriched in HCs from the Tg(myo6b:RiboTag) RNA-Seq using RT-qPCR in independent $\mathrm{HC}$ immunoprecipitation (IP) and input (IN) samples. Error bars represent fold change \pm standard deviation, and statistical significance was assessed by two-tailed Welch's $t$-test $(n=3)$. ${ }^{\star} p$-value $<0.05$, ${ }^{* *} p$-value $<0.01$, ${ }^{\star \star *} p$-value $<0.001$. (B) Validation of the same 10 highly enriched genes selected from the Tg(myo6b:RiboTag) RNA-Seq in sorted HCs by RT-qPCR. Black bar indicates the cycle threshold (CT) cutoff used to denote reliable expression (CT $\leq 30$ ). Error bars represent CT standard deviation between biological replicates $(n=3)$.

gene expression such as RT-qPCR and RNA-Seq. Additionally, this protocol is able to enrich for the zebrafish $\mathrm{HC}$ translatome, resulting in marked increases in transcript abundance of known $\mathrm{HC}$ expressed genes in the IP samples compared to RNA extracted from whole larvae (IN). Indeed, our RNA-Seq analysis of immunoprecipitated RNA shows enrichment for genes related to inner ear and lateral line $\mathrm{HC}$ function, while also showing depletion of genes involved in the function of other organs. Analysis of significantly enriched genes by RT-qPCR revealed high expression of genes not previously validated as expressed in zebrafish HCs, such as loxhd1a, loxhd1b, and dynlrb2, among others. Loxhd1a and loxhd1b are both homologs of the human deafness gene LOXHD1, the causative gene of DFNB77 (Grillet et al., 2009). Additionally, dynlrb2, a gene that encodes for a dynein light chain protein, has been previously identified as 
highly expressed in mouse HCs at both the transcript and protein levels, although its function in HCs has yet to be elucidated (Jiang et al., 2001; Scheffer et al., 2015; Shen et al., 2015; Hickox et al., 2017). Zebrafish may therefore be an appropriate model to study the roles that these genes play in normal HC function. Overall, taking into account the considerations outlined above, we believe that the $\operatorname{Tg}$ (myo6b:RiboTag) zebrafish represent an easy to use, versatile and sensitive model for studying inner ear and lateral line $\mathrm{HC}$ gene expression.

\section{AUTHOR CONTRIBUTIONS}

$\mathrm{AB}$ and $\mathrm{KK}$ generated the model, MM, YO, KK, and $\mathrm{RH}$ designed and interpreted the experiments, MM, AB, YO, and NP performed the experiments, YS analyzed the gene expression data, and $\mathrm{MM}, \mathrm{KK}$, and $\mathrm{RH}$ wrote the manuscript.

\section{FUNDING}

This work was supported by NIDCD/NIH R01DC013817 and R01DC03544 (RH), NIDCD/NIH Training Grant

\section{REFERENCES}

Anders, S., and Huber, W. (2010). Differential expression analysis for sequence count data. Genome Biol. 11:R106. doi: 10.1186/gb-2010-11-10-r106

Anders, S., Pyl, P. T., and Huber, W. (2015). Genome analysis HTSeq - a Python framework to work with high-throughput sequencing data. Bioinformatics 31, 166-169. doi: 10.1093/bioinformatics/btu638

Blanco-Sánchez, B., Clément, A., Fierro, A. Jr., Washbourne, P., and Westerfield, M. (2014). Complexes of Usher proteins preassemble at the endoplasmic reticulum and are required for trafficking and ER homeostasis. Dis. Models Mech. 7, 547-559. doi: $10.1242 / \mathrm{dmm} .014068$

Burns, J. C., Kelly, M. C., Hoa, M., Morell, R. J., and Kelley, M. W. (2015). Singlecell RNA-Seq resolves cellular complexity in sensory organs from the neonatal inner ear. Nat. Commun. 6:8557. doi: 10.1038/ncomms9557

Chang-Chien, J., Yen, Y. C., Chien, K. H., Li, S. Y., Hsu, T. C., Yang, J. J., et al. (2014). The connexin 30.3 of zebrafish homologue of human connexin 26 may play similar role in the inner ear. Hear. Res. 313, 55-66. doi: $10.1016 /$ j.heares.2014.04.010

Chatterjee, P., Padmanarayana, M., Abdullah, N., Holman, C. L., LaDu, J., Tanguay, R. L., et al. (2015). Otoferlin deficiency in zebrafish results in defects in balance and hearing: rescue of the balance and hearing phenotype with fulllength and truncated forms of mouse otoferlin. Mol. Cell. Biol. 35, 1043-1054. doi: 10.1128/MCB.01439-14

Di Donato, V., Auer, T. O., Duroure, K., and Del Bene, F. (2013). Characterization of the calcium binding protein family in zebrafish. PLOS ONE 8:e53299. doi: 10.1371/journal.pone.0053299

Elkon, R., Milon, B., Morrison, L., Shah, M., Vijayakumar, S., Racherla, M., et al. (2015). RFX transcription factors are essential for hearing in mice. Nat. Commun. 6:8549. doi: 10.1038/ncomms9549

Erickson, T., and Nicolson, T. (2015). Identification of sensory hair-cell transcripts by thiouracil-tagging in zebrafish. BMC Genomics 16:842. doi: 10.1186/s12864-015-2072-5

Erickson, T., Morgan, C. P., Olt, J., Hardy, K., Busch-Nentwich, E., Maeda, R., et al. (2017). Integration of Tmc1/2 into the mechanotransduction complex in zebrafish hair cells is regulated by 3 Transmembrane O-methyltransferase (Tomt). Elife 5, 1-26. doi: 10.7554/eLife.28474

Fang, M., Adams, J. S., McMahan, B. L., Brown, R. J., and Oxford, J. T. (2010). The expression patterns of minor fibrillar collagens during development in zebrafish. Gene Exp. Patterns 10, 315-322. doi: 10.1016/j.gep.2010.07.002
DC00046 (MM), NIDCD/NIH F31DC016218 (MM), and NIDCD/NIH Intramural Research Program Grant 1ZIADC000 085-01 (KK)

\section{ACKNOWLEDGMENTS}

The authors would like to thank Dr. David Raible from the University of Washington, as well as Dr. Doris Wu from the NIDCD, for their helpful comments on the manuscript. They would also like to thank Dr. Amiel A. Dror for contributing to the design of Figure 2A, Dr. Brant Weinstein and Dr. Mayumi Miller at NICHD for providing the RiboTag clone, as well as Dr. Xiaoxuan Fan, director of the University of Maryland School of Medicine Flow Cytometry Facility (SOM/UMGCC), for his help and expertise with the FACS experiments.

\section{SUPPLEMENTARY MATERIAL}

The Supplementary Material for this article can be found online at: https://www.frontiersin.org/articles/10.3389/fcell.2018. 00047/full\#supplementary-material

Gay, L., Karfilis, K. V., Miller, M. R., Doe, C. Q., and Stankunas, K. (2014). Applying thiouracil tagging to mouse transcriptome analysis. Nat. Protoc. 9, 410-420. doi: 10.1038/nprot.2014.023

Gay, L., Miller, M. R., Ventura, P. B., Devasthali, V., Vue, Z., Thompson, H. L., et al. (2013). Mouse TU tagging: a chemical/genetic intersectional method for purifying cell type-specific nascent RNA. Genes Dev. 27, 98-115. doi: $10.1101 /$ gad.205278.112

Gleason, M. R., Nagiel, A., Jamet, S., Vologodskaia, M., López-Schier, H., Hudspeth, A. J., et al. (2009). The transmembrane inner ear (Tmie) protein is essential for normal hearing and balance in the zebrafish. Proc. Natl. Acad. Sci. U.S.A. 106, 21347-21352. doi: 10.1073/pnas.0911632106

Grillet, N., Schwander, M., Hildebrand, M. S., Sczaniecka, A., Kolatkar, A., and Velasco, J., et al. (2009). Mutations in LOXHD1, an evolutionarily conserved stereociliary protein, disrupt hair cell function in mice and cause progressive hearing loss in humans. Am. J. Hum. Genet. 85, 328-337. doi: 10.1016/j.ajhg.2009.07.017.

Harris, M., Clark, J., Ireland, A., Lomax, J., Ashburner, M., Foulger, R., et al. (2004). The Gene Ontology (GO) database and informatics resource. Nucleic Acids Res. 32, 258-261. doi: 10.1093/nar/gkh036

Heiman, M., Schaefer, A., Gong, S., Peterson, J., Day, M., Ramsey, K. E., et al. (2009). Development of a BACarray translational profiling approach for the molecular characterization of CNS cell types. Cell 14, 738-748. doi: $10.1016 /$ j.cell.2008.10.028

Hertzano, R., and Elkon, R. (2012). High throughput gene expression analysis of the inner ear. Hear. Res. 288, 77-88. doi: 10.1016/j.heares.2012.01.002

Hickox, A. E., Wong, A. C. Y., Park, K., Strojny, C., Ramirez, M., Yates, J. R., et al. (2017). Cellular/molecular global analysis of protein expression of inner ear hair cells, J. Neurosci. 37, 1320-1339. doi: 10.1523/JNEUROSCI.2267-16.2016

Howe, D. G., Bradford, Y. M., Conlin, T., Eagle, A. E., Fashena, D., Frazer, K., et al. (2013). ZFIN, the Zebrafish Model Organism Database : increased support for mutants and transgenics. Nucleic Acids Res. 41, 854-860. doi: $10.1093 /$ nar/gks938

Jiang, J., Yu, L., Huang, X., Chen, X., Li, D., Zhang, Y., et al. (2001). Identification of two novel human dynein light chain genes, DNLC2A and DNLC2B, and their expression changes in hepatocellular carcinoma tissues from 68 Chinese patients. Gene 281, 103-113. doi: 10.1016/S0378-1119(01)00787-9

Jiang, L., Romero-Carvajal, A., Haug, J. S., Seidel, C. W., and Piotrowski, T. (2014). Gene-expression analysis of hair cell regeneration in the zebrafish lateral line. Proc. Natl. Acad. Sci. U.S.A. 111, E1383-E1392. doi: 10.1073/pnas.1402898111 
Kim, D., Pertea, G., Trapnell, C., Pimentel, H., Kelley, R., and Salzberg, S. L. (2013). TopHat2: accurate alignment of transcriptomes in the presence of insertions, deletions and gene fusions. Genome Biol. 14:R36. doi: 10.1186/gb-2013-14-4-r36

Kindt, K. S., Finch, G., and Nicolson, T. (2012). Kinocilia mediate mechanosensitivity in developing zebrafish hair cells. Dev. Cell 23, 329-341. doi: 10.1016/j.devcel.2012.05.022

Kozlowski, D. J., Whitfield, T. T., Hukriede, N. A., Lam, W. K., and Weinberg, E. S. (2005). The zebrafish dog-eared mutation disrupts eya1, a gene required for cell survival and differentiation in the inner ear and lateral line. Dev. Biol. 227, 27-41. doi: 10.1016/j.ydbio.2004. 08.033

Kwan, K. M., Fujimoto, E., Grabher, C., Mangum, B. D., Hardy, M. E., Campbell, D. S., et al. (2007). The Tol2kit: a multisite gateway-based construction kit forTol2 transposon transgenesis constructs. Dev. Dyn. 236, 3088-3099. doi: $10.1002 /$ dvdy.21343

Lieschke, G. J., and Currie, P. D. (2007). Animal models of human disease: zebrafish swim into view. Nat. Rev. 8, 353-367. doi: 10.1038/nrg2091

Maeda, R., Kindt, K. S., Mo, W., Morgan, C. P., Erickson, T., Zhao, H.,et al. (2014). Tip-link protein protocadherin 15 interacts with transmembrane channel-like proteins TMC1 and TMC2. Proc. Natl. Acad. Sci. U.S.A. 111, 12907-12912. doi: 10.1073/pnas.1402152111

Matern, M., Vijayakumar, S., Margulies, Z., Milon, B., Song, Y., Elkon, R., et al. (2017). GfilCre mice have early onset progressive hearing loss and induce recombination in numerous inner ear non-hair cells. Sci. Rep. 7:42079. doi: $10.1038 /$ srep42079

McDermott, B. M., Baucom, J. M., and Hudspeth, A. J. (2007). Analysis and functional evaluation of the hair-cell transcriptome. Proc. Natl. Acad. Sci. U.S.A. 104, 11820-11825. doi: 10.1073/pnas.0704476104

Millimaki, B. B., Sweet, E. M., Dhason, M. S., and Riley, B. B. (2007). Zebrafish atoh1 genes: classic proneural activity in the inner ear and regulation by Fgf and Notch. Development 134, 295-305. doi: 10.1242/ dev.02734

Mudunuri, U., Che, A., Yi, M., and Stephens, R. M. (2009). bioDBnet: the biological database network. Bioinformatics 25, 555-55610. doi: 10.1093/bioinformatics/btn654

Nicolson, T. (2005). The genetics of hearing and balance in zebrafish. Ann. Rev. Genet. 39, 9-22. doi: 10.1146/annurev.genet.39.073003.105049

Nicolson, T. (2017). The genetics of hair-cell function in zebrafish. J. Neurogenet. 31, 102-112. doi: 10.1080/01677063.2017.1342246

Obholzer, N., Wolfson, S., Trapani, J. G., Mo, W., Nechiporuk, A., BuschNentwich, E., et al. (2008). Cellular/molecular vesicular glutamate transporter 3 is required for synaptic transmission in zebrafish hair cells. J. Neurosci. 28, 2110-2118. doi: 10.1523/JNEUROSCI.5230-07.2008

Prykhozhij, S. V., Marsico, A., and Meijsing, S. H. (2013). Zebrafish Expression Ontology of Gene Sets (ZEOGS): a tool to analyze enrichment of zebrafish anatomical terms in large gene sets. Zebrafish 10, 303-315. doi: 10.1089/zeb.2012.0865

Ramsköld, D., Wang, E. T., Burge, C. B., and Sandberg, R. (2009). An abundance of ubiquitously expressed genes revealed by tissue transcriptome sequence data. PLoS Comput. Biol. 5:e1000598. doi: 10.1371/journal.pcbi.10 00598

Roh, H. C., Tsai, L. T.-Y., Lyubetskaya, A., Tenen, D. E., Kumari, M., Rosen, E., et al. (2017). Simultaneous transcriptional and epigenomic profiling from specific cell types within heterogeneous tissues in vivo. Cell Rep. 18, 1048-1061. doi: 10.1016/j.celrep.2016.12.087
Sanz, E., Yang, L., Su, T., Morris, D. R., McKnight, G. S., and Amieux, P. S. (2009). Cell-type-specific isolation of ribosome-associated mRNA from complex tissues. Proc. Natl. Acad. Sci. U.S.A. 106, 13939-13944. doi: 10.1073/pnas.0907143106

Scheffer, I., Shen, J., Corey, D. P., and Chen, Z. Y. (2015). Gene expression by mouse inner ear hair cells during development. J. Neurosci. 35, 6366-6380. doi: 10.1523/JNEUROSCI.5126-14.2015

Seiler, C., Ben-David, O., Sidi, S., Hendrich, O., Rusch, A., Burnside, B. et al. (2004). Myosin VI is required for structural integrity of the apical surface of sensory hair cells in zebrafish. Dev. Biol. 272, 328-338. doi: 10.1016/j.ydbio.2004.05.004

Seiler, C., Finger-Baier, K. C., Rinner, O., Makhankov, Y. V., Schwarz, H., Neuhauss, S. C., et al. (2005). Duplicated genes with split functions: independent roles of protocadherin 15 orthologues in zebrafish hearing and vision. Dev. Dis. 132, 615-623. doi: 10.1242/dev.01591

Shen, J., Scheffer, D. I., Kwan, K. Y., and Corey, D. P. (2015). SHIELD: an integrative gene expression database for inner ear research. Database 2015:bav071. doi: 10.1093/database/bav071

Shen, Y.-C., Jeyabalan, A. K., Wu, K. L., Hunker, K. L., Kohrman, D. C., Thompson, D. L., et al. (2008). The transmembrane inner ear (tmie) gene contributes to vestibular and lateral line development and function in the zebrafish (Danio rerio). Dev. Dyn. 237, 941-952. doi: 10.1002/dvdy.21486

Steiner, A. B., Kim, T., Cabot, V., and Hudspeth, A. J. (2014). Dynamic gene expression by putative hair-cell progenitors during regeneration in the zebrafish lateral line. Proc. Natl. Acad. Sci. U.S.A. 111, E1393-E1401. doi: 10.1073/pnas.1318692111

Tao, L., DeRosa, A. M., White, T. W., and Valdimarsson, G. (2010). Zebrafish cx30.3: identification and characterization of a gap junction gene highly expressed in the skin. Dev. Dyn. 239, 2627-2636. doi: 10.1002/dvdy.22399

Thisse, B., and Thisse, C. (2004). Fast Release Clones: A High Throughput Expression Analysis., ZFIN Direct Data Submission.

Tryon, R. C., Pisat, N., Johnson, S. L., and Dougherty, J. D. (2013). Development of translating ribosome affinity purification for zebrafish. Genesis 192, 187-192. doi: $10.1002 /$ dvg. 22363

Van Camp, G., and Smith, R. (2017). Hereditary Hearing Loss Homepage. Available online at: http://hereditaryhearingloss.org

van den Brink, S. C., Sage, F., Vértesy, Á., Spanjaard, B., and Peterson-Maduro, J., Baron, C. S., et al. (2017). Single-cell sequencing reveals dissociationinduced gene expression in tissue subpopulations. Nat. Methods 14, 935-936. doi: $10.1038 /$ nmeth.4437

Wang, L., Sewell, W. F., Kim, S. D., Shin, J. T., MacRae, C. A., Zon, L. I., et al. (2008). Eya4 regulation of $\mathrm{NA}+/ \mathrm{K}+$-ATPase is required for sensory development in zebrafish. Development 135, 3425-3434. doi: $10.1242 /$ dev.012237

Conflict of Interest Statement: The authors declare that the research was conducted in the absence of any commercial or financial relationships that could be construed as a potential conflict of interest.

Copyright (c) 2018 Matern, Beirl, Ogawa, Song, Paladugu, Kindt and Hertzano. This is an open-access article distributed under the terms of the Creative Commons Attribution License (CC BY). The use, distribution or reproduction in other forums is permitted, provided the original author(s) and the copyright owner(s) are credited and that the original publication in this journal is cited, in accordance with accepted academic practice. No use, distribution or reproduction is permitted which does not comply with these terms. 